\title{
Dietary patterns among British adults in 2000/2001: associations with salt consumption and macronutrient intakes
}

\author{
S. Gibson ${ }^{1}$ and M. Ashwell ${ }^{2}$ \\ ${ }^{1}$ Sig-Nurture Ltd., 11 Woodway, Guildford, Surrey GU1 2TF, UK and ${ }^{2}$ Ashwell Associates Ltd, Ashwell Street, Ashwell, \\ Hertfordshire SG7 5PZ, UK
}

Analysis of dietary patterns using multivariate techniques takes account of the multi-dimensional nature of food habits as practised in the population. It also offers the opportunity to explore whether patterns are compatible with other dietary guidelines such as those for saturated fat and sugar. This study used principal component analysis (PCA) to identify dietary patterns and their association with salt and macronutrient intake in the National Diet and Nutrition Survey: adults aged 19 to 64 years ${ }^{(1)}$, using data from 1724 adults completing $7 \mathrm{~d}$ weighed food records in 2000/2001.

Adults were classified as low-salt consumers $(<6 \mathrm{~g} / \mathrm{d})$, average consumers $(6-8 \mathrm{~g} / \mathrm{d})$ or high- salt consumers $(8+\mathrm{g} / \mathrm{d})$ for preliminary analysis. High salt intakes were attributable to eating more food in general and to a higher sodium density of the diet (mg/MJ energy). Some foods (notably processed meat products and bread) contributed large amounts of sodium, and were consumed in higher proportion $(\mathrm{g} / \mathrm{MJ})$ by those with high salt intakes. Conversely, low-salt consumers $(<6 \mathrm{~g} / \mathrm{d})$ ate more vegetables, fruit and fish, and drank more beverages $(\mathrm{g} / \mathrm{MJ})$. Increased consumption of salt was associated with diets of higher energy density, higher in fat and saturates $(\%$ energy) but lower in non-milk extrinsic sugars (NMES). Bread contributed more than $21 \%$ of salt intake and meat products approximately $15 \%$, while ready-to-eat breakfast cereals (i.e. excluding porridge) contributed $4.2 \%$ in $2000 / 2001$.

PCA revealed 8 patterns (PC1 to PC8), which explained $40 \%$ of the total variance in food intakes. PC3 (with high loadings on bread, fats and cheese) was positively associated with a high salt intake $(r=0.6)$ and sodium dense diet $(r=0.2)$. Two further patterns were associated with high salt intake: 'red meat, sauces and alcohol', (PC7) $(r=0.38)$ and 'meat products, eggs and chips' (PC2) $(r=0.32)$, which was also high in fat and saturates (SFA). By contrast, the 'health-conscious' pattern (PC1) (vegetables, fruit, fruit juice and fish), the 'breakfast cereals and milk' pattern (PC6) and a 'chicken and rice' pattern (PC8) were associated with a modest salt intake, lower sodium density and lower fat and SFA. Other patterns were 'tea/coffee and cakes' (PC4) (associated with high SFA and NMES) and 'soft drinks and snacks' (pizza, pasta and crisps) (PC5). Patterns with high loading on meat products (PC2 and PC7) were correlated with a declared practice of usually adding salt to food, while PC1 and PC6 were associated with infrequent salt use.

Since this survey was conducted, substantial reductions in the salt content of many processed products have been achieved, including a $44 \%$ reduction in sodium content of breakfast cereals ${ }^{(2)}$. New NDNS data for 2008/9 suggest that salt intake from food now averages 6 g/d for adults ${ }^{(3)}$; although excretion averages $\sim 8.6 \mathrm{~g} / \mathrm{d}^{(4)}$, suggesting discretionary salt use is still a significant contributor. We suggest that dietary advice to lower salt consumption, which is broadly consistent with dietary guidelines for saturated fats, should also take account of wider nutritional attributes of foods and the varying dietary habits and energy needs of consumers.

Data was obtained from the UK Data Archive (http://www.data-archive.ac.uk). This analysis was supported by a financial contribution from Kellogg's. Initial set-up work was funded by the Food Standards Agency as part of project N08023.

1. Henderson L, Irving K, Gregory J et al. (2003) The National Diet and Nutrition Survey: adults aged 19 to 64 years. Volume 3: Vitamin and Mineral Intake and Urinary Analytes. TSO, London: Office for National Statistics.

2. Food Standards Agency (2009). Salt reduction Targets. http://www.food.gov.uk/healthiereating/salt/saltreduction

3. Food Standards Agency (2010). Headline results from Year 1 of the rolling programme 2008/9 http://www.food.gov.uk/science/dietarysurveys/ ndnsdocuments/ndns0809year1

4. Food Standards Agency (2008). Dietary sodium levels surveys. http://www.food.gov.uk/science/dietarysurveys/urinary 